Association for

Computing Machinery

Advancing Computing as a Science \& Profession

\title{
PPoPP'12
}

Proceedings of the 2012 ACM SIGPLAN Symposium on Principles and Practice of Parallel Programming

Sponsored by:

ACM SIGPLAN 
Association for

Computing Machinery

Advancing Computing as a Science \& Profession

\author{
The Association for Computing Machinery \\ 2 Penn Plaza, Suite 701 \\ New York, New York 10121-0701
}

Copyright (C) 2012 by the Association for Computing Machinery, Inc. (ACM). Permission to make digital or hard copies of portions of this work for personal or classroom use is granted without fee provided that copies are not made or distributed for profit or commercial advantage and that copies bear this notice and the full citation on the first page. Copyright for components of this work owned by others than ACM must be honored. Abstracting with credit is permitted. To copy otherwise, to republish, to post on servers or to redistribute to lists, requires prior specific permission and/or a fee. Request permission to republish from: Publications Dept., ACM, Inc. Fax +1 (212) 869-0481 or<permissions@acm.org>.

For other copying of articles that carry a code at the bottom of the first or last page, copying is permitted provided that the per-copy fee indicated in the code is paid through the Copyright Clearance Center, 222 Rosewood Drive, Danvers, MA 01923 (USA).

Notice to Past Authors of ACM-Published Articles

ACM intends to create a complete electronic archive of all articles and/or other material previously published by ACM. If you have written a work that has been previously published by ACM in any journal or conference proceedings prior to 1978, or any SIG Newsletter at any time, and you do NOT want this work to appear in the ACM Digital Library, please inform permissions@acm.org, stating the title of the work, the author(s), and where and when published.

ISBN: 978-1-4503-1160-1

Additional copies may be ordered prepaid from:

\title{
ACM Order Department
}

PO Box 30777

New York, NY 10087-0777, USA

Phone: 1-800-342-6626 (USA and Canada)

+1-212-626-0500 (Global)

Fax: +1-212-944-1318

E-mail: acmhelp@acm.org

Hours of Operation: 8:30 am - 4:30 pm ET

ACM Order Number: 551121

Printed in the USA 


\title{
Chairs' Welcome
}

It is our great pleasure to welcome you to the 17th ACM SIGPLAN Symposium on Principles and Practice of Parallel Programming (PPoPP'12). PPoPP continues its tradition of serving as a leading forum for research in all aspects of parallel software, including theoretical foundations, programming models, algorithms, applications, and systems software. With the ubiquity of parallelism in commodity processors and the increasing use of GPUs for high-performance computing, the effective use of parallel systems is being recognized as one of the most challenging problems faced today.

PPoPP'12 received 173 complete paper submissions. In addition to the 25 program committee members, 75 members of the external review committee provided reviews for the papers. Two rounds of reviewing were conducted, with at least three reviews being obtained in the first round, with additional reviews being obtained in the second round for papers where needed. After extensive discussions at an in-person two-day program committee meeting in November 2011, 26 full papers were selected for presentation at the conference. PPoPP' 12 continues the tradition of poster presentations of high quality submissions that could not be accepted as full papers. This year's conference features 32 poster presentations over two sessions.

PPoPP'12 is again co-located this year with the International Symposium on High-Performance Computer Architecture (HPCA), allowing attendees of one conference the option of attending talks at the other. We feature two joint HPCA/PPOPP keynote presentations. Sanjeev Kumar from Facebook will present a keynote on "Social Networking at Scale," and Keshav Pingali from the University of Texas at Austin will present a keynote entitled "Parallel Programming Needs Data-Centric Foundations.”

PPoPP' 12 is a result of the efforts of a large number of people. Our thanks, first and foremost, to the authors of all the submissions, the keynote speakers, and workshop and tutorial presenters, for providing the intellectual content of the program. We are grateful for the tireless efforts of the program committee and external review committee, and the program subchairs: Xiaoming Li, publicity and web; Xipeng Shen, workshops and tutorials; Sriram Krishnamoorthy, publications; David Koppelman, local arrangements; Gerald Baumgartner, finance; John Cavazos, registration. We are grateful for the support provided by Carrie Stein, Karen Jones and a number of volunteers. We thank Luis Ceze for making arrangements for the program committee meeting on the campus of the University of Washington, and Rich Gerber for exceptional assistance with the START conference management software. We are grateful to Lisa Tolles and the team at Sheridan Printing for an efficient and streamlined publication process. Finally, our thanks to ACM SIGPLAN for its ongoing sponsorship; to the steering committee (Keshav Pingali, Calin Cascaval, Mary Hall, David Padua, and Pen Yew) for their guidance; and to our partners for their financial support.

\author{
P. (Saday) Sadayappan \\ J. (Ram) Ramanujam \\ PPoPP'12 Program Chair \\ PPoPP'12 General Chair \\ The Ohio State University, USA \\ Louisiana State University, USA
}





\section{Table of Contents}

PPoPP 2012 Conference Organization ix

\section{Session 1: GPU Tools}

- Scalable Framework for Mapping Streaming Applications Onto Multi-GPU Systems . 1 Huynh Phung Huynh ( $A$ *STAR Institute of High Performance Computing), Andrei Hagiescu, Weng-Fai Wong (National University of Singapore), Rick Siow Mong Goh ( ${ }^{*}$ STAR Institute of High Performance Computing)

- A Performance Analysis Framework for Identifying Potential Benefits in GPGPU Applications .... 11 Jaewoong Sim, Aniruddha Dasgupta, Hyesoon Kim, Richard Vuduc (Georgia Institute of Technology)

- Efficient Performance Evaluation of Memory Hierarchy for Highly Multithreaded Graphics Processors Sara S. Baghsorkhi, Isaac Gelado, Matthieu Delahaye, Wen-mei W. Hwu (University of Illinois at Urbana-Champaign)

\section{Session 2: Communication \& SIMD Optimization}

- Communication Avoiding Symmetric Band Reduction Grey Ballard, James Demmel, Nicholas Knight (University of California, Berkeley)

- Faster Topology-Aware Collective Algorithms Through Non-minimal Communication .... 45 Paul Sack, William Gropp (University of Illinois at Urbana-Champaign)

- Efficient SIMD Code Generation for Irregular Kernels. .55 Seonggun Kim (Samsung Advanced Institute of Technology), Hwansoo Han (Sungkyunkwan University)

- Extending a C-like Language for Portable SIMD Programming Roland Leißa, Sebastian Hack (Saarland University), Ingo Wald (Intel Corporation)

\section{Session 3: Programming Models}

- A Hybrid Approach of OpenMP for Clusters Okwan Kwon, Fahed Jubair, Rudolf Eigenmann, Samuel Midkiff (Purdue University)

- DOJ: Dynamically Parallelizing Object-Oriented Programs. Yong hun Eom, Stephen Yang, James C. Jenista, Brian Demsky (University of California, Irvine)

- S: A Scripting Language for High-Performance RESTful Web Services Daniele Bonetta, Achille Peternier, Cesare Pautasso, Walter Binder (University of Lugano)

\section{Session 4: GPU Algorithms}

- A GPU Implementation of Inclusion-Based Points-to Analysis Mario Méndez-Lojo (University of Texas), Martin Burtscher (Texas State University), Keshav Pingali (University of Texas)

- Scalable GPU Graph Traversal

Duane Merrill (University of Virginia), Michael Garland (NVIDIA Corporation), Andrew Grimshaw (University of Virginia)

- GPU-based NFA Implementation for Memory Efficient High Speed Regular Expression Matching Yuan $\mathrm{Zu}$, Ming Yang, Zhonghu Xu, Lin Wang, Xin Tian, Kunyang Peng, Qunfeng Dong (University of Science and Technology of China)

\section{Session 5: Concurrent Data Structures}

- A Methodology for Creating Fast Wait-Free Data Structures 141 Alex Kogan, Erez Petrank (Technion, Israel Institute of Technology) 
- Concurrent Tries with Efficient Non-Blocking Snapshots

Aleksandar Prokopec (École Polytechnique Fédérale de Lausanne), Nathan G. Bronson (Stanford University),

Phil Bagwell (Typesafe), Martin Odersky (École Polytechnique Fédérale de Lausanne)

- A Speculation-Friendly Binary Search Tree

Tyler Crain (IRISA), Vincent Gramoli (École Polytechnique Fédérale de Lausanne), Michel Raynal (IRISA)

- PARRAY: A Unifying Array Representation for Heterogeneous Parallelism

Yifeng Chen, Xiang Cui, Hong Mei (Peking University)

\section{Session 6: Parallel Algorithms}

- Internally Deterministic Parallel Algorithms Can Be Fast

Guy E. Blelloch (Carnegie Mellon University), Jeremy T. Fineman (Georgetown University),

Phillip B. Gibbons (Intel Labs), Julian Shun (Carnegie Mellon University)

- Deterministic Parallel Random-Number Generation for Dynamic-Multithreading Platforms

Charles E. Leiserson, Tao B. Schardl, Jim Sukha (Massachusetts Institute of Technology),

- Scalable Parallel Minimum Spanning Forest Computation

Sadegh Nobari, Thanh-Tung Cao (National University of Singapore), Panagiotis Karras (Rutgers University),

Stéphane Bressan (National University of Singapore)

\section{Session 7: Correctness and Fault Tolerance}

- GKLEE: Concolic Verification and Test Generation for GPUs.

Guodong Li (Fujitsu Laboratories of America),

Peng Li, Geof Sawaya, Ganesh Gopalakrishnan (University of Utah),

Indradeep Ghosh, Sreeranga P. Rajan (Fujitsu Labs of America)

- Algorithm-based Fault Tolerance for Dense Matrix Factorizations

Peng Du, Aurelien Bouteiller, George Bosilca, Thomas Herault, Jack Dongarra

(University of Tennessee, Knoxville)

- Efficient Deadlock Avoidance for Streaming Computation with Filtering

Jeremy D. Buhler, Kunal Agrawal, Peng Li, Roger D. Chamberlain (Washington University in St. Louis)

\section{Session 8: Scheduling and Synchronization}

- Lock Cohorting: A General Technique for Designing NUMA Locks

David Dice, Virendra J. Marathe (Oracle Labs),

Nir Shavit (Massachusetts Institute of Technology \& Tel-Aviv University)

- Revisiting the Combining Synchronization Technique...

Panagiota Fatourou (University of Crete \& FORTH ICS), Nikolaos D. Kallimanis (University of Ioannina)

- A Work-Stealing Scheduler for X10's Task Parallelism with Suspension Olivier Tardieu (IBM T.J. Watson Research Center),

Haichuan Wang (University of Illinois at Urbana-Champaign), Haibo Lin (IBM Research - China)

\section{Poster Session 1 (Monday)}

- Automatic Communication Optimizations Through Memory Reuse Strategies

Muthu Baskaran, Nicolas Vasilache, Benoit Meister, Richard Lethin (Reservoir Labs Inc.)

- FlexBFS: A Parallelism-Aware Implementation of Breadth-First Search on GPU

Gu Liu, Hong An, Wenting Han, Xiaoqiang Li, Tao Sun, Wei Zhou, Xuechao Wei, Xulong Tang

(University of Science and Technology of China)

- Programming Parallel Embedded and Consumer Applications in OpenMP Superscalar

Michael Andersch, Chi Ching Chi, Ben Juurlink (Technische Universität Berlin)

- An Overview of Medusa: Simplified Graph Processing on GPUs.

Jianlong Zhong, Bingsheng He (Nanyang Technological University)

- Optimizing Remote Accesses for Offloaded Kernels: Application to High-Level Synthesis for FPGA

Christophe Alias, Alain Darte, Alexandru Plesco (UCB-Lyon) 
- Using GPU's to Accelerate Stencil-based Computation Kernels for the Development of Large Scale Scientific Applications on Heterogeneous Systems.

Jian Tao (Louisiana State University), Marek Blazewicz (Poznań Supercomputing and Networking Center),

Steven R. Brandt (Louisiana State University)

- Mechanizing the Expert Dense Linear Algebra Developer

Bryan Marker, Robert van de Geijn, Don Batory (The University of Texas at Austin),

Andy Terrel (Texas Advanced Computing Center), Jack Poulson (The University of Texas at Austin)

- The Boat Hull Model: Adapting the Roofline Model to Enable Performance Prediction for Parallel Computing.

Cedric Nugteren, Henk Corporaal (Eindhoven University of Technology)

- Speculative Parallelization on GPGPUs

Min Feng, Rajiv Gupta, Laxmi N. Bhuyan (University of California, Riverside)

- Adapting the Polyhedral Model as a Framework for Efficient Speculative Parallelization Alexandra Jimborean, Philippe Clauss, Benoît Pradelle, Luis Mastrangelo, Vincent Loechner (INRIA \& LSIIT \& University of Strasbourg)

- An Overview of CMPI: Network Performance Aware MPI in the Cloud

Yifan Gong, Bingsheng He, Jianlong Zhong (Nanyang Technological University)

- OpenCL as a Unified Programming Model for Heterogeneous CPU/GPU Clusters.

Jungwon Kim, Sangmin Seo, Jun Lee, Jeongho Nah, Gangwon Jo, Jaejin Lee (Seoul National University)

- BDDT: Block-Level Dynamic Dependence Analysis for Deterministic Task-Based Parallelism ......301 George Tzenakis, Angelos Papatriantafyllou, John Kesapides, Polyvios Pratikakis (FORTH),

Hans Vandierendonck (FORTH \& Ghent University), Dimitrios S. Nikolopoulos (FORTH)

- Portable Parallel Performance from Sequential, Productive, Embedded

Domain-Specific Languages

Shoaib Kamil, Derrick Coetzee, Scott Beamer, Henry Cook, Ekaterina Gonina

(University of California, Berkeley),

Jonathan Harper (Mississippi State University), Jeffrey Morlan, Armando Fox (University of California, Berkeley)

- Communication-Centric Optimizations by Dynamically Detecting Collective Operations .

Torsten Hoefler (University of Illinois at Urbana-Champaign),

Timo Schneider (Chemnitz University of Technology)

\section{Poster Session 2 (Tuesday)}

- LHlf: Lock-free Linear Hashing (poster paper)

Donghui Zhang (Microsoft Jim Gray Systems Lab), Per-Åke Larson (Microsoft Research)

- Wait-Free Linked-Lists

Shahar Timnat, Anastasia Braginsky, Alex Kogan, Erez Petrank (Technion, Israel Institute of Technology)

- Scalable Parallel Debugging with Statistical Assertions

Minh Ngoc Dinh, David Abramson, Chao Jin (Monash University),

Andrew Gontarek, Bob Moench, Luiz DeRose (Cray Inc.)

- Verification of Software Barriers

Alexander Malkis, Anindya Banerjee (IMDEA Software Institute),

- Collective Algorithms for Sub-communicators.

Anshul Mittal, Thomas George, Yogish Sabharwal (IBM Research India),

Nikhil Jain (University of Illinois at Urbana Champaign), Sameer Kumar (IBM T.J. Watson Research Center)

- Synchronization Views for Event-loop Actors Joeri De Koster, Stefan Marr, Theo D’Hondt (Vrije Universiteit Brussel)

- CPHASH: A Cache-Partitioned Hash Table.

Zviad Metreveli, Nickolai Zeldovich, M. Frans Kaashoek (Massachusetts Institute of Technology)

- RACECAR: A Heuristic for Automatic Function Specialization on Multi-core

Heterogeneous Systems

John R. Wernsing, Greg Stitt (University of Florida) 
- A Lock-Free, Array-Based Priority Queue

Yujie Liu, Michael Spear (Lehigh University)

- An Infrastructure for Dynamic Optimization of Parallel Programs Albert Noll, Thomas R. Gross (ETH Zurich)

- Automatic Datatype Generation and Optimization

Fredrik Kjolstad (Massachusetts Institute of Technology),

Torsten Hoefler, Marc Snir (University of Illinois at Urbana-Champaign)

- NDetermin: Inferring Nondeterministic Sequential Specifications for Parallelism Correctness .....329 Jacob Burnim, Tayfun Elmas, George Necula, Koushik Sen (University of California, Berkeley)

- Concurrent Breakpoints

Chang-Seo Park, Koushik Sen (University of California, Berkeley)

- Establishing a Miniapp as a Programmability Proxy

Andrew I. Stone (Colorado State University), John M. Dennis (National Center for Atmospheric Science), Michelle Mills Strout (Colorado State University)

- OpenMP-Style Parallelism in Data-Centered Multicore Computing with R. Lei Jiang (Louisiana State University), Pragneshkumar B. Patel (University of Tennessee), George Ostrouchov (Oak Ridge National Laboratory), Ferdinand Jamitzky (Leibniz Supercomputing Centre)

- Performance Analysis of Parallel Constraint-Based Local Search Yves Caniou (JFLI, CNRS / NII), Daniel Diaz (University of Paris 1-Sorbonne), Florian Richoux, Philippe Codognet (JFLI, CNRS / UPMC / University of Tokyo), Salvador Abreu (Universidade de Évora \& CENTRIA FCT/UNL) 


\section{PPOPP 2012 Conference Organization}

General Chair: J. Ramanujam (Louisiana State University, USA)

Program Chair: P. Sadayappan (The Ohio State University, USA)

Workshops and Tutorials Chair: Xipeng Shen (College of William and Mary, USA)

Proceedings Chair: Sriram Krishnamoorthy (Pacific Northwest National Laboratory, USA)

Local Arrangements Chair: David Koppelman (Louisiana State University, USA)

Web and Publicity Chair: Xiaoming Li (University of Delaware, USA)

Registration Chair: John Cavazos (University of Delaware, USA)

Finance Chair: Gerald Baumgartner (Louisiana State University, USA)

Steering Committee Chair: Keshav Pingali (University of Texas at Austin, USA)

Steering Committee: Calin Cascaval (Qualcomm, USA)

Mary Hall (University of Utah, USA)

David Padua (University of Illinois, USA)

Pen-Chung Yew (Academica Sinica, Taiwan \& University of Minnesota, USA)

Program Committee: Emery Berger (University of Massachusetts at Amherst, USA)

Uday Bondhugula (Indian Institute of Science, India)

Greg Bronevetsky (Lawrence Livermore National Laboratory, USA)

Luis Ceze (University of Washington, USA)

Brad Chamberlain (Cray, USA)

Sandhya Dwarkadas (University of Rochester, USA)

Rudi Eigenmann (Purdue University, USA)

Michael Garland (Nvidia, USA)

Rajiv Gupta (University of California at Riverside, USA)

Francois Irigoin (MINES ParisTech, France)

Sriram Krishnamoorthy (Pacific Northwest National Laboratory, USA)

Andrew Lumsdaine (Indiana University, USA)

John Mellor-Crummey (Rice University, USA)

Mario Mendez-Lojo (Advanced Micro Devices, USA)

Frank Mueller (North Carolina State University, USA)

Michael O'Boyle (University of Edinburgh, UK)

John Owens (University of California at Davis, USA)

Padma Raghavan (Pennsylvania State University, USA)

Vijay Saraswat (IBM Research, USA)

Martin Schulz (Lawrence Livermore National Laboratory, USA)

Xipeng Shen (College of William and Mary, USA) 
(continued): Michelle Strout (Colorado State University, USA)

Jeff Vetter (Oak Ridge National Laboratory and Georgia Tech., USA)

Sam Williams (Lawrence Berkeley National Laboratory, USA)

Jingling Xue (University of New South Wales, Australia)

External Review Committee: Gul Agha

Kunal Agrawal

Gagan Agrawal

Alex Aiken

George Almasi

Srinivas Aluru

Nancy Amato

Scott Baden

Martin Burtscher

Dhruva Chakrabarti

Barbara Chapman

Arun Chauhan

Albert Cohen

Bronis R. de Supinski

Steven Derrien

Jim Dinan

Thomas Fahringer

Cormac Flanagan

Guang Gao

Maria Garzaran

Ganesh Gopalakrishnan

Ramasamy Govindarajan

Naga Govindaraju

Ananth Grama

Paul Hargrove

Tim Harris

Yuxiong He

Torsten Hoefler

Wei-Chung Hsu

Costin Iancu

Roy Ju

Laxmikant Kale

Mahmut Kandemir

Jens Knoop

Milind Kulkarni

Jaejin Lee

Zhiyuan Li
Kamesh Madduri

Allen Malony

Virendra Marathe

Maged Michael

Sam Midkiff

Eliot Moss

Madanlal Musuvathi

Dimitrios Nikolopoulos

Vijay Pai

Scott Pakin

Santosh Pande

Srinivasan Parthasarathy

Erez Petrank

Rodric Rabbah

Sanjay Rajopadhye

Thomas Rauber

Lawrence Rauchwerger

Juan Carlos Saez

Marc Shapiro

Tatiana Shpeisman

Arrvindh Shriraman

Allan Snavely

Marc Snir

Michael Spear

Nathan Tallent

Kenjiro Taura

Michael Taylor

Philippas Tsigas

Christoph von Praun

Richard Vuduc

Peng Wu

Ayal Zaks

Binyu Zang

Antonia Zhai

Xiaodong Zhang

Huiyang Zhou 
Additional Reviewers: Sriram Ananthakrishnan

Corine Ancourt

Rajkishore Barik

Michael Bauer

Tom Bergan

Darius Buntinas

Antal Buss

Daniel Cederman

Milind Chabbi

Sunita Chandrasekaran

Minas Charalambides

Daniel Chavarria

Feng Chen

Wei-Fan Chiang

Fabien Coelho

Murray Cole

Tony Curtis

Joseph Devietti

Xiaoning Ding

Peter Dinges

Christophe Dubach

William E. Byrd

Nicholas Edmonds

Laura Effinger-Dean

James Elliott

Carl Evans

Xing Fang

Damon Fenacci

Chris Fensch

David Fiala

Justin Frye

Lokesh Gidra

Neal Glew

Dominic Grewe

Jayanth Gummaraju

Phuong Ha

Jeff Hammond

Albert Hartono

Eric Holk

Shantonu Hossain

Yin Huai

Khaled Ibrahim

OhYoung Jang

Gangwon Jo
Herbert Jordan

Pierre Jouvelot

Changhee Jung

Wookeun Jung

Jaeyeon Kang

Rajesh Karmani

Derrick Kearney

Jungwon Kim

Tobias von Koch

Christopher Krieger

Stephen Kyle

Michael Lai

Brian Larkins

Hugh Leather

Jae-woo Lee

Jun Lee

Rubao Lee

Sangho Lee

Guodong Li

Peng Li

Chu-Cheow Lim

Jean-Pierre Lozi

Binbin Lu

Brandon Lucia

Ravi Mangal

R. Manikantan

Sandya S. Mannarswamy

Joseph Manzano

Kirill Mechitov

Sébastien Monnet

Karthik Murthy

Vlad Nae

Jorge Navas

Dang Nhan Nguyen

Molly A. O’Neil

Adam Oliner

Daniel Orozco

Sreepathi Pai

Prasanna Pandit

Ioannis Papadopoulos

Chang-Seo Park

Simone Pellegrini

Antoniu Pop

Ashwin Prasad 
(continued): Andreas Prell

Gil Rapaport

Kaushik Ravichandran

Eugene Ressler

Olivier Rojo

Adrian Sampson

William Scherer III

Carsten Scholtes

Sangmin Seo

Shishir Sharma

Subodh Sharma

Reza Shiftehfar

Sunil Shrestha

Yingchong Situ

Timmie Smith

Moulik Soni

Abhishek Sreenivasa

Tom St. John

Jeff Stuart

Hakan Sundell

Peter Thoman

Nathan Thomas
Chen Tian

Kai Tian

Daniel Tomkins

Sean Treichler

Antonino Tumeo

Ioannis Venetis

Kaibo Wang

Zheng Wang

Jeremiah Willcock

Benjamin P. Wood

Alec $\mathrm{Wu}$

Xing $\mathrm{Wu}$

Xintian Yang

Yi Yang

Tomofumi Yuki

Mani Zandifar

Xiao Zhang

Yongpeng Zhang

Zheng Zhang

Yili Zheng

Christopher Zimmer

Stephane Zuckerman

Sponsor:

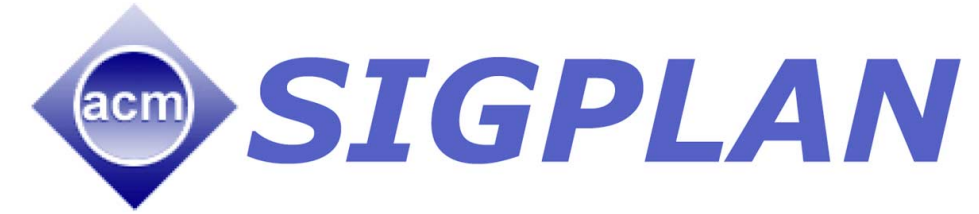

Supporters:

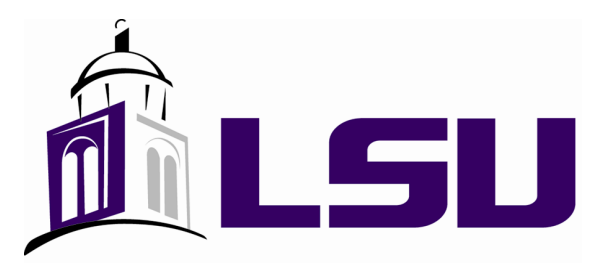

LOUISIANA STATE UNIVERSITY

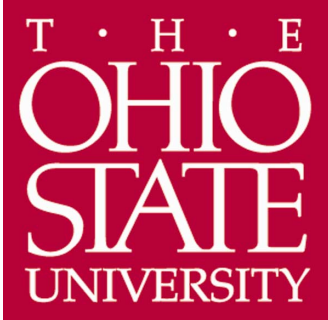

